\title{
Multidimensional subwavelength position sensing using a semiconductor laser with optical feedback
}

\author{
Seth D. Cohen, ${ }^{1, *}$ Andrés Aragoneses, ${ }^{2}$ Damien Rontani, ${ }^{1}$ M. C. Torrent, ${ }^{2}$ \\ Cristina Masoller, ${ }^{2}$ and Daniel J. Gauthier ${ }^{1}$ \\ ${ }^{1}$ Duke University, Physics Department, Box 90305, Durham, North Carolina 27708, USA \\ ${ }^{2}$ Departament de Física i Enginyeria Nuclear, Universitat Politecnica de Catalunya, Colom 11, 08222 Barcelona, Spain \\ *Corresponding author: sdc18@phy.duke.edu
}

Received July 22, 2013; revised September 10, 2013; accepted September 16, 2013;

posted September 19, 2013 (Doc. ID 194315); published October 21, 2013

\begin{abstract}
We demonstrate experimentally how to harness quasi-periodic dynamics in a semiconductor laser with dual optical feedback for measuring subwavelength changes in each arm of the cavity simultaneously. We exploit the multifrequency spectrum of quasi-periodic dynamics and show that independent frequency shifts are mapped uniquely to two-dimensional displacements of the arms in the external cavity. Considering a laser diode operating at telecommunication wavelength $\lambda \approx 1550 \mathrm{~nm}$, we achieve an average nanoscale resolution of approximately $9.8 \mathrm{~nm}$ $(\sim \lambda / 160)$. (c) 2013 Optical Society of America

OCIS codes: (280.3420) Laser sensors; (250.5960) Semiconductor lasers.

http://dx.doi.org/10.1364/OL.38.004331
\end{abstract}

The extreme sensitivity of semiconductor lasers with optical feedback has been studied for its applications to sensors [1]. For example, the subwavelength sensitivity to changes in the external cavity length of the feedback has triggered the development of laser-feedback interferometry, also referred as self-mixing interferometry [2-4] , where displacements and vibrations are measured with subwavelength resolution by tracking changes in the laser intensity. Other approaches have used changes in the relaxation-oscillation frequency [5]. These laser-based approaches usually lead to the extraction of a single observable, thus restricting sensing or imaging to $1 \mathrm{D}$. As a consequence, to image or sense complex objects in 2D or $3 \mathrm{D}$, such laser-based systems require scanning and sequential 1D acquisitions.

Recently, a subwavelength 2D position sensor was realized using a high-speed nonlinear feedback system comprising a radio-frequency $(\mathrm{RF})\left(\lambda \sim 10^{-2} \mathrm{~m}\right)$ wavechaotic cavity [6]. It was demonstrated that the $2 \mathrm{D}$ position of an object inside the cavity could be determined with subwavelength precision using a linear map that uniquely associates frequency shifts in the quasi-periodic spectrum of the dynamics with the object position [6]; this method does not require sequential scanning to deduce $2 \mathrm{D}$ information. An open question is whether these frequency shifts can also occur in the optical domain, thus allowing for the development of novel laserfeedback-based devices with the capability for subwavelength, nanoscale, multidimensional position sensing.

In this Letter, we demonstrate experimentally, using a semiconductor laser with quasi-periodic dynamics induced by external optical feedback from a cavity with two arms (referred to as the T-cavity), the existence of frequency shifts in the spectrum, similar to those found in [6]. We unveil a quadratic mapping between these frequency shifts with independent, subwavelength translations of each arm of the T-cavity. We show that our approach leads to a highly sensitive detection scheme for 2D nanoscale translations.

Our experimental setup is shown in Fig. 1. A singlemode semiconductor laser operating at $\lambda=1550.8 \mathrm{~nm}$ is subjected to dual optical feedback with two arms of lengths $x$ and $y$, respectively. The propagation distances along each path result in two different time delays, $\tau_{x} \sim 55.5 \mathrm{~ns}$ and $\tau_{y} \sim 55.6 \mathrm{~ns}$, with feedback strengths $\eta_{x, y}$ approximately equal and controlled simultaneously by a single variable optical attenuator placed before the beam splitter (see Fig. 1). A polarization controller is used to ensure coherent feedback to the laser (verified using L-I curves [7]). Our setup is reminiscent of previous experiments with T-cavity optical feedback [8-10].

The relative values of the time delays are controlled using two piezoelectric transducers $\left(\mathrm{PZT}_{x, y}\right)$ that move the positions of the mirrors on a nanoscale by $\Delta_{x} \ll x$ and $\Delta_{y} \ll y$, respectively. To estimate the transducers' displacements as a function of the applied voltages, we

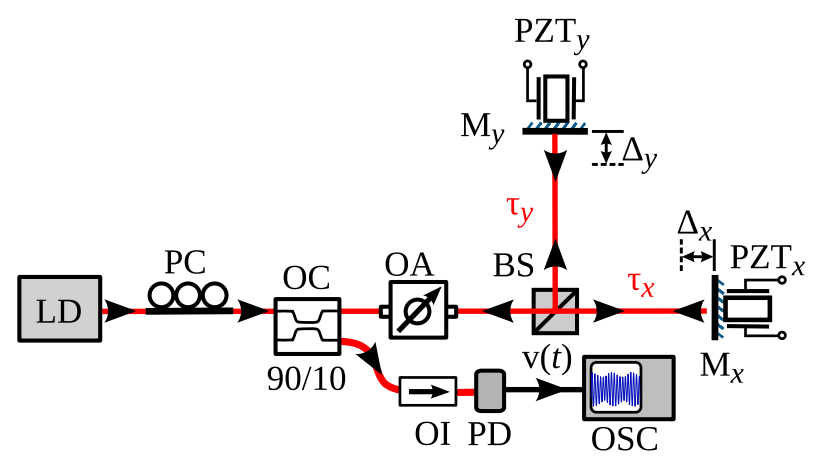

Fig. 1. Two-delay all-optical feedback system for multidimensional subwavelength sensing. The output of a laser diode (LD, Sumitomo SLT4416-DP) passes through a polarization control (PC), a 90/10 optical coupler (OC), and an optical attenuator (OA), and separates using a 50/50 beam splitter (BS). The feedback strength is set so that it reduces the laser threshold by $\sim 1 \%$. The optical field propagates along two separate paths with time delays $\tau_{x, y}$. Piezoelectric transducers $\left(\mathrm{PZT}_{x, y}\right.$, Burleigh PZO-015) translate mirrors $\mathrm{M}_{x, y}$ by $\Delta_{x, y}$ and change feedback delay $\tau_{x, y}$. After an optical isolator (OI), detection is performed using an ac-coupled $12 \mathrm{GHz}$ photodetector (PD, New Focus 1544-B) and a $40 \mathrm{GS} / \mathrm{s}, 8 \mathrm{GHz}$ analog bandwidth, high-speed digital oscilloscope (OSC, Agilent DSO90804A). 
calibrate them via interferometry. Based on our calibration measurements, the translations per applied voltages to $\mathrm{PZT}_{x, y}$ are $29.2 \pm 0.1 \mathrm{~nm} / \mathrm{V}$ and $45.8 \pm 0.2 \mathrm{~nm} / \mathrm{V}$, respectively. We note that this calibration does not include either the hysteresis or the nonlinear response of the PZTs under applied voltages. To reduce the effects of the PZT nonlinearity and hysteresis, we bias them at $50 \mathrm{~V}$ (out of a total range of 0-150 V) and scan them by at most $3 \mathrm{~V}$ about this value. Based on the manufacturer's specification, we estimate that the deviation from linearity is at most $0.3 \%$ for our experiment and hence we ignore these behaviors in the analysis described below.

We carefully adjust the feedback strength such that the optical intensity displays quasi-periodic oscillations in time. This occurs when the feedback strength is about $\sim 2 \%$, consistent with previous reports of feedbackinduced quasi-periodicity $[11,12]$. A typical optical intensity time series is displayed in Fig. 2(a). It shows a fast oscillatory signal with slow modulation of its amplitude. The frequency spectrum of the quasi-periodic signal is given in Fig. 2(b). Within the $8 \mathrm{GHz}$ bandwidth of our oscilloscope, it reveals multiple clusters of frequency peaks ranging from $\sim 1$ to $\sim 7 \mathrm{GHz}$ (additional clusters above $8 \mathrm{GHz}$ may be present but are not visible without suitable measurement equipment). The fourth cluster concentrates the largest amount of spectral power because it lies in the vicinity of the relaxation-oscillation frequency measured to be $\sim 6.5 \mathrm{GHz}$ for pumping current $I=23.6 \mathrm{~mA}$. The peak in the $i$ th cluster with the maximum spectral power is denoted by $f_{i}$. In Figs. 2(c) and $2(\mathrm{~d})$, we show zoomed regions on the spectrum,
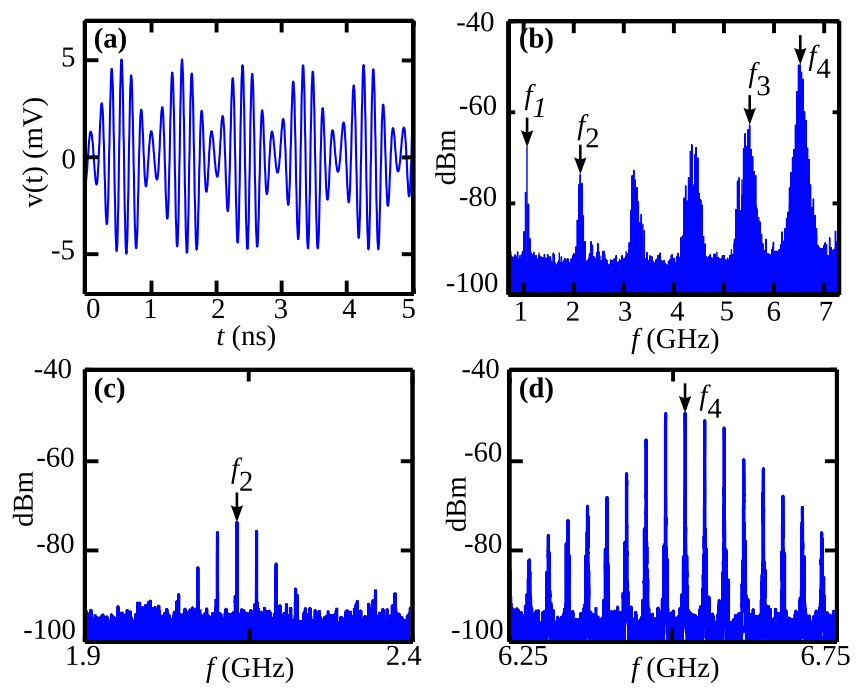

Fig. 2. Experimental quasi-periodic dynamics generated by a semiconductor laser with dual optical feedback. (a) Temporal evolution of $v(t)$ generated by the photodetector and proportional to the intensity of the optical field. The pump current is $I=23.6 \mathrm{~mA}$ (the threshold is $I_{\mathrm{th}} \sim 8.0 \mathrm{~mA}$ ), $\tau_{x} \approx 55.5$, $\tau_{y} \approx 55.6 \mathrm{~ns}$ (corresponding to $\Delta_{x, y}=0$ ), and $\sim 2 \%$ of optical intensity fed back in the laser cavity. (b) Power spectral density (PSD) of $v(t)$ in the quasi-periodic regime showing four frequency clusters, each labeled by their central frequency $f_{i}, i=1, \ldots, 4\left(f_{1}=1.07 \mathrm{GHz}, f_{2}=2.13 \mathrm{GHz}, f_{3}=5.42 \mathrm{GHz}\right.$, and $f_{4}=6.49 \mathrm{GHz}$ ). (c), (d) Zooms on the structures of the second and fourth labeled frequency clusters later used for subwavelength sensing. highlighting $f_{2}$ and $f_{4}$, respectively. We investigate the relative frequency shifts $\Delta f_{i}$ to the values of $f_{i}$ due to subwavelength translations $\Delta_{x, y} \ll \lambda$ of each arm of the dual optical feedback. These translations induce changes in the time delays denoted by $\Delta \tau_{x, y}=2 \Delta_{x, y} / c$, where $c$ is the speed of light in free space.

We track the frequencies of the quasi-periodicity as $\tau_{x, y}$ are changed simultaneously by translating the positions of mirrors $\mathrm{M}_{x, y}$. For this, we use computer-controlled, high-voltage power supplies connected to $\mathrm{PZT}_{x, y}$. The piezoelectric transducers are translated by steps of $\sim 10 \mathrm{~nm}$ within an approximative $100 \mathrm{~nm} \times 100 \mathrm{~nm} 2 \mathrm{D}$ grid. Over this subwavelength region of interest, the dynamics remain quasi-periodic. Over substantially larger regions, we observe that the dynamics bifurcate to a new behavior or undergo frequency hopping. At each point of the grid, we measure the frequency shifts $\Delta f_{i}$ associated with each frequency $f_{i}$ of the four labeled clusters.

The nanoscale modification to the cavity induced by the transducer leads to observed frequency shifts $\Delta f_{i}$ on a kilohertz scale, which is orders of magnitude smaller compared to frequencies $f_{i}$. To resolve such fine variations, we use the trigger skew on our digital oscilloscope, so that the amount of time that the oscilloscope waits between its initial triggering and the acquisition of a waveform is $t_{\text {skew }}=5 \mu \mathrm{s}$. Using a large trigger skew causes small frequency shifts $\Delta f_{i}$ to be observed as visible phase shifts $\Delta \varphi_{i}=\Delta f_{i} \cdot t_{\text {skew }}$ across the oscilloscope's delayed acquisition window.

Using this approach, measurements of $\Delta f_{i}$ have a resolution set by the jitter of the waveform. To reduce the effect of jitter, we acquire (for each point of the 2D grid) a waveform $\bar{v}(t)$ resulting from the average of 500 time series. The averaging is realized in real time using the oscilloscope. We find that averaging over 500 time-series measurements optimizes the trade-off between noise reduction in the measurement and the inevitable drift in the apparatus due to temperature fluctuations. Better thermal shielding in future experiments will minimize this nonideal behavior. To successfully average over quasiperiodic waveforms, the trigger height $v_{\text {trig }}$ must also be tuned so that the oscilloscope triggers only on the maxima of the largest amplitudes, corresponding to regions of the quasi-periodic signal for which the incommensurate frequencies add constructively. Other triggering values, such as $v_{\text {trig }}=0 \mathrm{~V}$, will cause the averaged waveforms to collapse to zero because of the nonperiodic nature of the quasi-periodic signal. Using a calibrated quasi-periodic waveform generated by a stable function generator (Agilent E8267D with $10^{-3} \mathrm{~Hz}$ frequency resolution), our frequency-resolving protocol yields a $\pm 3 \mathrm{kHz}$ frequency resolution.

We monitor the quasi-periodic frequency shifts $\Delta f_{i}$ as a function of $\left(\Delta_{x}, \Delta_{y}\right)$. We approximate them using a nonlinear least-squares regression to a model of a four-tone quasi-periodic signal of the averaged data from the delayed acquisition window of the oscilloscope

$$
\bar{v}(t) \approx \sum_{1 \leq i \leq 4} A_{i} \sin \left[2 \pi\left(f_{i}+\Delta f_{i}\right)\left(t-t_{\text {skew }}\right)\right]
$$


where $A_{i}$ and $\Delta f_{i}$ are the parameters estimated via regression and $f_{i}$ is measured directly with the oscilloscope to achieve approximately $100 \mathrm{kHz}$ resolution (using time series with $\sim 5.25 \times 10^{5}$ points).

As an example, we plot $\Delta f_{2}$ and $\Delta f_{4}$ as a function of $\left(\Delta_{x}, \Delta_{y}\right)$ over the entire 2D position grid in Figs. 3(a) and $3(\mathrm{~b})$. Interestingly, we do not observe a near-planar evolution of the frequency shifts similar to those observed in [6]. Our two-delay optical system produces frequency shifts that are mapped onto a smooth manifold with nonzero curvature.

The two manifolds exhibit different ranges of variations and levels of curvature, thus suggesting independent (and thus invertible) frequency shifts as a function of the PZT's translations. To demonstrate independence and inversion, we propose to fit the nonlinear map $\left(\Delta f_{2}, \Delta f_{4}\right) \rightarrow\left(\Delta_{x}, \Delta_{y}\right)$ by quadratic bivariate functions

$$
\Delta f_{2,4}\left(\Delta_{x}, \Delta_{y}\right)=\sum_{0 \leq i+j \leq 2} a_{i j \mid 2,4} \Delta_{x}^{i} \Delta_{y}^{j}
$$

where the values of the fitted coefficients $a_{i j \mid 2,4}$ obtained via regression are given in the caption of Fig. 3 . The fitted functions of Eq. (2) are numerically invertible so that predicted values for $\Delta_{x, y}$ can be related to a given measure of frequency shifts $\Delta f_{2,4}$. The root-mean-square differences between the predicted and actual subwavelength translations realized by $\mathrm{PZT}_{x, y}$ are 12.3 and $6.8 \mathrm{~nm}$, respectively. This gives an average resolution of $9.8 \mathrm{~nm} \sim \lambda / 160$. The maximum errors between the experimental map and the fit are $\varepsilon_{x, \max }=27.5 \mathrm{~nm}$ $(\sim \lambda / 60)$ and $\varepsilon_{y, \text { max }}=22.7 \mathrm{~nm}(\sim \lambda / 70)$, which sets a bound on the achievable resolution. In our experiments, the frequency shifts associated with $\Delta f_{2,4}$ yield the best $2 \mathrm{D}$ resolution. A lower bound for the dynamic range for our system, given by the ratio between the observed range and resolution, is $\sim 10$ on the $100 \mathrm{~nm} \times 100 \mathrm{~nm}$ grid. [As we define it, the dynamic range is $\sim 100 \mathrm{~nm}$
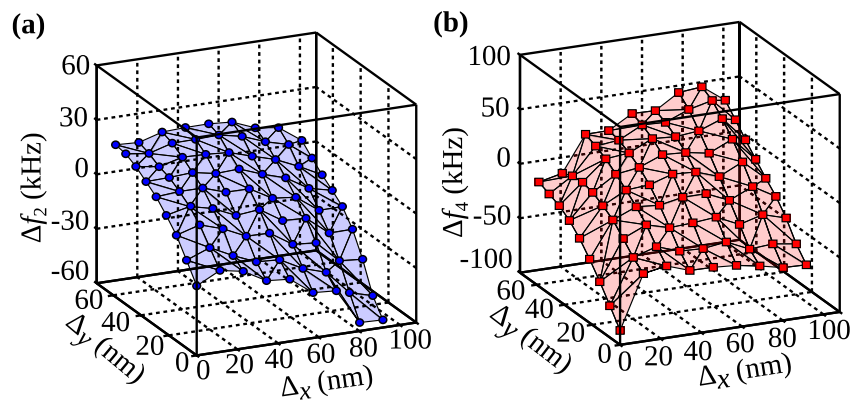

Fig. 3. Experimental manifold associated with the quasiperiodic frequency shifts (a) $\Delta f_{2}$ and (b) $\Delta f_{4}$ as a function of $\Delta_{x, y}$. The 2D manifolds are fitted with quadratic functions with coefficients for (a) $a_{00 \mid 2}=-17.3784 \pm 1.0834 \mathrm{kHz}, a_{01 \mid 2}=$ $-0.0691 \pm 0.0358 \mathrm{kHz} \cdot \mathrm{nm}^{-1}, a_{10 \mid 2}=1.2458 \pm 0.0562 \mathrm{kHz} \cdot \mathrm{nm}^{-1}$, $a_{11 \mid 2}=0.0058 \pm 0.0005 \mathrm{kHz} \cdot \mathrm{nm}^{-2}, a_{02 \mid 2}=-0.0041 \pm 0.0003$ $\mathrm{kHz} \cdot \mathrm{nm}^{-2}$, and $a_{20 \mid 2}=-0.0095 \pm 0.0009 \mathrm{kHz} \cdot \mathrm{nm}^{-2} ; \quad$ and (b) $\quad a_{00 \mid 4}=-67.7941 \pm 3.2683 \mathrm{kHz}, \quad a_{01 \mid 4}=1.2050 \pm 0.1079$ $\mathrm{kHz} \cdot \mathrm{nm}^{-1}, a_{10 \mid 4}=1.8789 \pm 0.1694 \mathrm{kHz} \cdot \mathrm{nm}^{-1}, a_{11 \mid 4}=0.0093 \pm$ $0.0014 \mathrm{kHz} \cdot \mathrm{nm}^{-2}, \quad a_{02 \mid 4}=-0.0127 \pm 0.0010 \mathrm{kHz} \cdot \mathrm{nm}^{-2}$, and $a_{20 \mid 4}=-0.0140 \pm 0.0026 \mathrm{kHz} \cdot \mathrm{nm}^{-2}$. (maximum observed range in each direction) $/ \sim 10 \mathrm{~nm}$ (resolution).]

We conjecture that room temperature fluctuations (which are not controlled in our experiment) are the main sources of errors in our measurements. They induce erratic changes of the path lengths of each arm in the cavity, thus affecting the frequencies $f_{i}$ with parasitic frequency shifts. Hence, the manifolds displayed in Fig. 3 can be used only once as calibration measurements to then infer an unknown subwavelength trajectory on the $100 \mathrm{~nm} \times 100 \mathrm{~nm}$ grid. Within a $\sim 5$ min time period, which corresponds to the typical time scale for the drift in our apparatus, we realize back-to-back acquisitions of 81 calibration points followed by 32 trajectory points. Using the calibration points, we realize the mapping to then recover the shape of the trajectory with an average $2 \mathrm{D}$ resolution of $\sim \lambda / 160$ (for details, see [13]). Beyond the 5 min time window, temperature fluctuations prevent us from inferring arbitrary subwavelength positions from the calibration.

In summary, we have presented the foundation for realizing a 2D position sensor with nanometer resolution using a quasi-periodic optical field generated by a semiconductor laser with optical feedback. To the best of our knowledge, this is the first experimental study of a T-cavity optical feedback for subwavelength multidimensional position sensing based on frequency shifts in the quasi-periodic dynamics spectrum. In [6], Cohen et al. hypothesize that their technique for subwavelength position sensing may be scalable to the nanoscale using optical feedback. Our work shows that the physical mechanism responsible for subwavelength sensing in the RF domain, independent quasi-periodic frequency shifts, remains true in the optical domain, thus laying the foundation for an all-optical implementation of their setup.

We envision several future directions for this dynamical phenomenon. Similar to []ㅡ, the delayed feedback of the optical system can be coupled into a wave-chaotic optical cavity [14] such that it interacts with a contained subwavelength scatterer. In its present form, our system may also be used as a multidimensional sensor similar to those developed for monitoring of chemical concentrations [15] or nanoparticles [16]. Last, the Hopf frequency of a semiconductor laser with feedback has been studied with respect to the external cavity length [11]; developing theories for the quasi-periodic frequencies in a T-cavity subject to small delay changes may give deeper insight into our observations.

S. D. C., D. R., and D. J. G. gratefully acknowledge the financial support of the U.S. Office of Naval Research grant no. N000014-07-0734. A. A., M. C. T., and C. M. gratefully acknowledge the financial support of grant no. FA8655-12-1-2140 from EOARD, grant no. FIS201237655-C02-01 from the Spanish MCI, and grant no. 2009 SGR 1168 from the Generalitat de Catalunya. C. M. also acknowledges support from the ICREA Academia program.

\section{References}

1. J. Ohtsubo, Semiconductor Lasers: Stability, Instability and Chaos (Springer, 2006). 
2. S. Donati, G. Giuliani, and S. Merlo, IEEE J. Quantum Electron. 31, 113 (1995).

3. D. M. Kane and K. A. Shore, Unlocking Dynamical Diversity: Optical Feedback Effects in Semicondustor Lasers (Wiley, 2005), Chap. 7.

4. U. Zabit, R. Atashkhooei, T. Bosch, S. Royo, F. Bony, and A. D. Rakic, Opt. Lett. 35, 1278 (2010).

5. E. Lacot, R. Day, J. Pinel, and F. Stoeckel, Opt. Lett. 26, 1483 (2001).

6. S. Cohen, H. Cavalcante, and D. J. Gauthier, Phys. Rev. Lett. 107, 254103 (2011).

7. J. Mink and B. H. Verbeek, Appl. Phys. Lett. 48, 745 (1986).

8. Y. Liu and J. Ohtsubo, Opt. Lett. 19, 448 (1994).

9. M. J. Bunner, A. Kittel, J. Parisi, I. Fischer, and W. Elsäßer, Europhys. Lett. 42, 353 (1998).
10. F. Rogister, D. W. Sukow, A. Gavrielides, P. Mégret, O. Deparis, and M. Blondel, Opt. Lett. 25, 808 (2000).

11. J. Mørk, B. Tromborg, and J. Mark, IEEE J. Quantum Electron. 28, 93 (1992).

12. C. Masoller, Phys. Rev. A 50, 2569 (1994).

13. S. D. Cohen, "Subwavelength sensing using nonlinear feedback in a wave-chaotic cavity," Ph.D. thesis (Duke University, 2013).

14. T. Gensty, K. Becker, I. Fischer, W. Elsäßer, C. Degen, P. Debernardi, and G. P. Bava, Phys. Rev. Lett. 94, 233901 (2005).

15. B. E. De Martini, J. F. Rhoads, M. A. Zielke, K. G. Owen, S. W. Shaw, and K. L. Turner, Appl. Phys. Lett. 93, 054102 (2008).

16. H.-Y. Lin, C.-H. Huang, C.-C. Huang, Y.-C. Liu, and L.-K. Chau, Opt. Lett. 37, 3969 (2012). 